\title{
Effects of Hydrogen Bonding on the Monolayer Properties of Amphiphilic \\ Double-decker Shaped Polyhedral Silsesquioxanes
}

Asuman C. Kucuk, Jun Matsui, Tokuji Miyashita

Institute of Multidisciplinary Research for Advanced Materials (IMRAM), Tohoku University, 2-1-1

Katahira, Aoba-ku, Sendai 980-8577, Japan

\section{SUPPORTING INFORMATION}

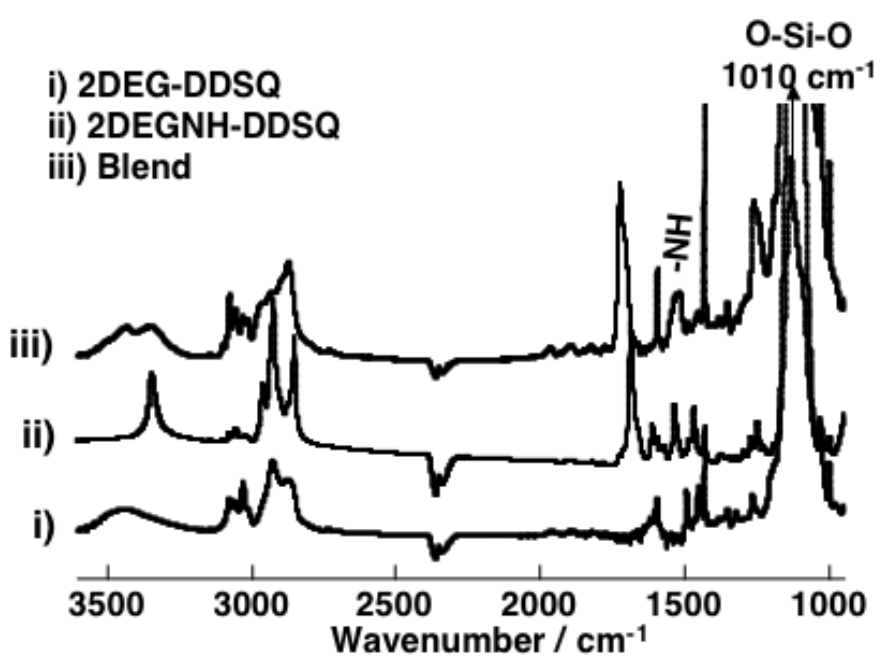

Figure S1 FT-IR spectra of (a) 2DEG-DDSQ, (b) 2DEGNH-DDSQ and (c) their 1:1 mixture. 


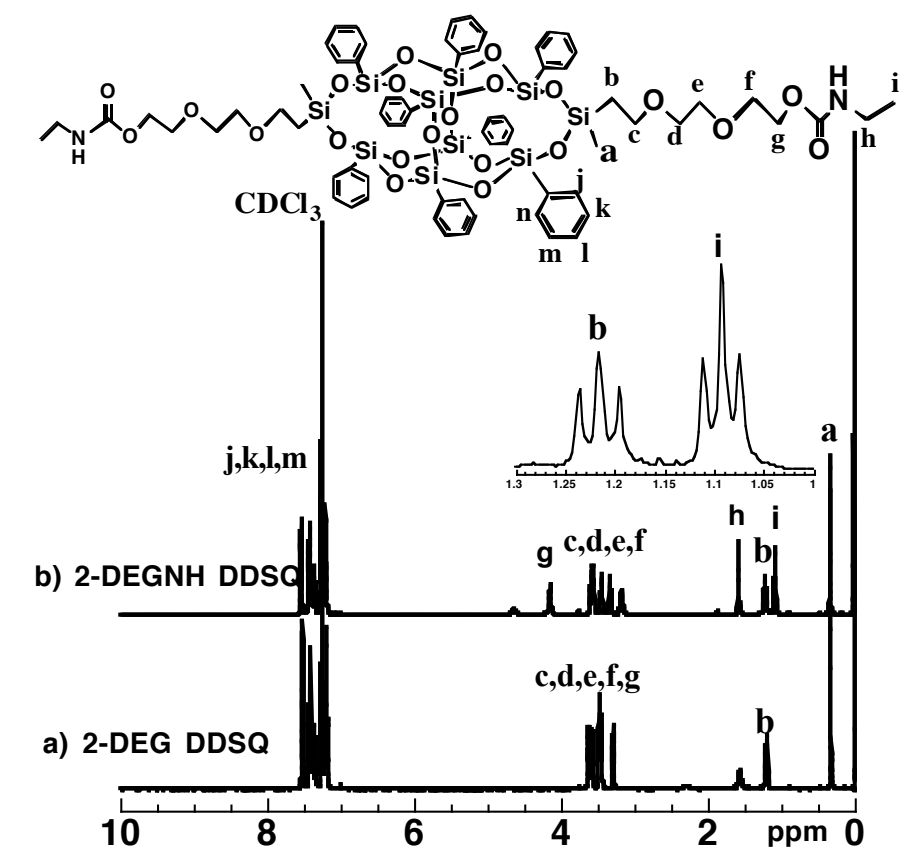

Figure S2 ${ }^{1} \mathrm{H}$ NMR spectra of (a) 2DEG-DDSQ, (b) 2DEGNH-DDSQ.

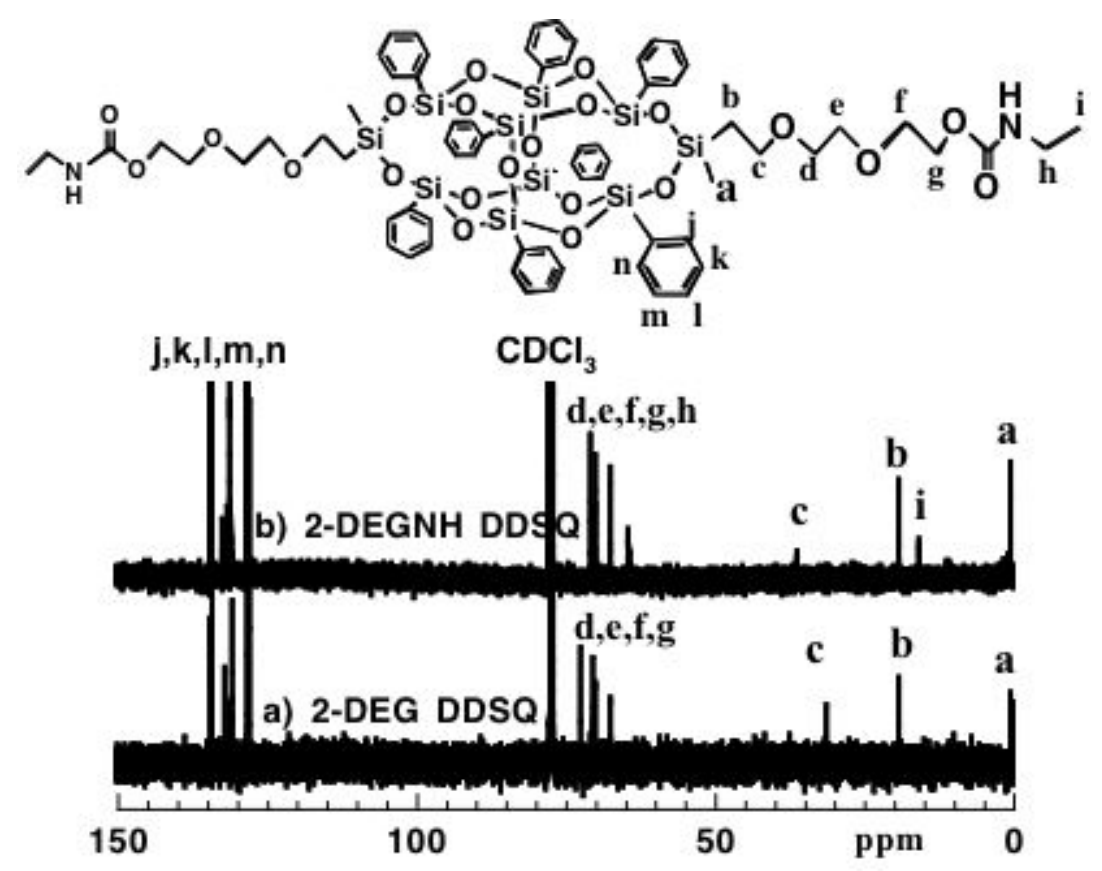

Figure S3 ${ }^{13} \mathrm{C}$ NMR spectra of (a) 2DEG-DDSQ, (b) 2DEGNH-DDSQ. 